\title{
CRITERIA FOR RATING THE LEVEL AND QUALITY OF DIGITALIZATION OF THE EDUCATIONAL PROCESS IN UNIVERSITIES OF THE RUSSIAN FEDERATION
}

\author{
Elena V. Brodovskaya \\ Financial University under the Government of the Russian Federation, Moscow, Russian Federation; \\ Moscow State Pedagogical University, Moscow, Russian Federation \\ Anna Yu. Dombrovskaya \\ Moscow State Pedagogical University, Moscow, Russian Federation; \\ Financial University under the Government of the Russian Federation, Moscow, Russian Federation \\ Roman V. Pyrma \\ Financial University under the Government of the Russian Federation, Moscow, Russian Federation
}

\section{Artur A. Azarov}

Financial University under the Government of the Russian Federation, Moscow, Russian Federation

Annotation. Introduction. The article reflects the authors' system for calculating the level and quality of digitalization of the educational process in Russian universities. This task is actualized by a number of circumstances of modern realities. The need to create a digital twin / double for each university has become a trend due to the importance of providing applicants, students and graduates with the most accessible educational environment. The high-quality digital environment of a university during the spread of pandemics is an important condition for maintaining the stability of the educational process. Today, the ability of universities to quickly adapt the digital educational space to the needs of distance learning is a significant sign of the viability and quality of the management staff of higher education institutions. Methods and materials. To develop the system, the authors used a mathematical tool - the method of summary indicators for calculating the level of digitalization of universities, which allows to evaluate various characteristics of the value, efficiency and quality of objects with a significant number of different parameters. Analysis. To develop indicators - basic rating indicators - the authors used the results of a preliminary study of the best practices of digitalization of the university environment of leading foreign and Russian universities. The system takes into account the needs for digitalization of the educational environment of three different categories: Tु applicants, students and graduates. Results. The introduction of the rating into the Russian practice will allow to justify a system of recommendations to university administrations to improve the level and quality of their digitalization of the educational process.

Key words: digitalization, educational environment, rating, quality criteria, method of summary indicators.

Citation. Brodovskaya E.V., Dombrovskaya A.Yu., Pyrma R.V., Azarov A.A. Criteria for Rating the Level and Quality of Digitalization of the Educational Process in Universities of the Russian Federation. Vestnik Volgogradskogo gosudarstvennogo universiteta. Seriya 4. Istoriya. Regionovedenie. Mezhdunarodnye otnosheniya [Science Journal of Volgograd State University. History. Area Studies. International Relations], 2020, vol. 25, no. 2, pp. 268-283. (in Russian). DOI: https://doi.org/10.15688/jvolsu4.2020.2.20 


\section{КРИТЕРИИ ДЛЯ РЕЙТИНГОВАНИЯ УРОВНЯ И КАЧЕСТВА ЦИФРОВИЗАЦИИ ПРОЦЕССА ОБРАЗОВАНИЯ В ВУЗАХ РФ}

\section{Елена Викторовна Бродовская}

Финансовый университет при Правительстве Российской Федерации, г. Москва, Российская Федерация; Московский педагогический государственный университет, г. Москва, Российская Федерация

\section{Анна Юрьевна Домбровская}

Московский педагогический государственный университет, г. Москва, Российская Федерация; Финансовый университет при Правительстве Российской Федерации, г. Москва, Российская Федерация

\section{Роман Васильевич Пырма}

Финансовый университет при Правительстве Российской Федерации, г. Москва, Российская Федерация

\section{Артур Александрович Азаров}

Финансовый университет при Правительстве Российской Федерации, г. Москва, Российская Федерация

Аннотация. Введение. В статье отражается авторская система расчета уровня и качества цифровизации процесса образования в вузах РФ. Данная задача актуализируется рядом обстоятельств современных реалий. Ставшая трендовой необходимость создания для каждого университета цифрового двойника обусловлена значимостью предоставления абитуриентам, студентам и выпускникам максимально доступной образовательной среды. Качественная цифровая среда вуза во время распространения пандемий - важное условие сохранения стабильности образовательного процесса. Сегодня способность университетов оперативно приспособить цифровое образовательное пространство под нужны дистанционного обучения - значимый признак состоятельности и качества их управленческого аппарата. Meтоды и материальl. Для разработки системы привлечен математический аппарат - метод сводных показателей для расчета уровня цифровизации вузов. Он позволяет оценивать различные характеристики ценности, эффективности и качества объектов, имеющих существенное количество различных параметров. Анализ. Для разработки индикаторов - базовых показателей рейтингования - использованы результаты предварительного исследования передового опыта цифровизации вузовской среды ведущих зарубежных и российских университетов. Результаты. Система учитывает потребности в цифровизации образовательной среды трех разных категорий: абитуриентов, студентов и выпускников. Внедрение рейтинга в российскую практику позволит обосновать систему рекомендаций администрациям университетов по повышению уровня и качества осуществляемой ими цифровизации процесса образования. Вклад авторов. Е.В. Бродовская, являясь автором идеи создания публикации, осуществляла общее научное руководство работой исследовательского коллектива и научное редактирование аналитического текста. А.Ю. Домбровская реализовала направление анализа, связанное с систематизацией критериев рейтингования. А.А. Азаров обеспечил привлечение математического аппарата в процессе создания системы рейтингования вузов по качеству и уровню цифровизации образовательной среды. Р.В. Пырма привлек широкую теоретико-методологическую базу для реализации обзорной части статьи и обоснования теоретического фундамента публикации. Общим фронтом работы соавторов стали актуализация темы, подготовка ее постановочного раздела, осуществление методологической триангуляции в процессе осмысления результатов анализа и подведения итогов научного исследования.

Ключевые слова: цифровизация, образовательная среда, рейтинг, критерии качества, метод сводных показателей.

Цитирование. Бродовская Е. В., Домбровская А. Ю., Пырма Р. В., Азаров А. А. Критерии для рейтингования уровня и качества цифровизации процесса образования в вузах РФ // Вестник Волгоградского государственного университета. Серия 4, История. Регионоведение. Международные отношения. - 2020. - Т. 25 , № 2. - C. 268-283. - DOI: https://doi.org/10.15688/jvolsu4.2020.2.20 
Введение. Повышение качества цифровых образовательных сред учреждений высшего образования является одной из значимых задач, от успешности решения которой во многом зависят процессы цифровизации экономики, формирования культуры цифрового гражданства, адаптации общества к структурным изменениям рынка труда в России. При условии, что нашу страну можно отнести к группе государств «второй волны» распространения Интернета, благодаря чему число российских онлайн-пользователей составляет на сегодняшний день более 90 миллионов человек, состояние цифровых образовательных сред университетов отражает ряд особенностей. Среди них можно выделить следующие: цифровое неравенство (по поколенческому и компетентностному признакам), вторичность цифровых образовательных технологий, недостаточность инфраструктуры цифровых образовательных сред и др. Кроме того, система мониторинга учреждений высшего образования в России не включает ни одного параметра, связанного с оценкой качества цифровых образовательных сред. Это в свою очередь лишает вузы стимулов совершенствовать инфраструктуру и качество цифровых коммуникаций в образовательном процессе. Мы исходим из понимания того, что одним из механизмов решения обозначенных проблем может стать построение рейтинга качества цифровизации образовательных сред в университетах России.

Появление мировых рейтингов вузов отражает тенденцию глобализации высшего образования и растущий интерес к сопоставлению его качества [9]. При этом международные рейтинги университетов сравнивают не только конкурентные преимущества учреждений, но и показывают уровень развития национальных систем образования. Некоторые исследователи связывают рост значения мировых рейтингов вузов с их превращением в инструмент конкурентной борьбы и образовательной политики [1]. Рейтинги влияют на государственную политику, выбор студентов и их семей, поскольку воспринимаются как мера качества и создают таким образом интенсивную конкуренцию между университетами по всему миру.

По мнению Э. Хейзелкорн, так как рейтинги университетов отвечают на запрос сту- дентов, родителей, политиков, работодателей и других заинтересованных сторон в отношении объективной информации о качестве образования, они приобрели высокое значение в мире, став существенным фактором, формирующим институциональную репутацию [16]. Университеты и правительства все чаще используют их для повышения своего статуса, привлечения иностранных студентов, преподавателей и инвестиций, а во многих случаях устанавливают политику, направленную на улучшение положения в рейтинге, что создает как позитивные основания конкуренции, так и «порочные стимулы» соответствия требованиям [22]. Плюсы и минусы классификации университетов подробно представлены в исследовании ЮНЕСКО «Рейтинги и ответственность в высшем образовании: использование и злоупотребления» [22].

Рейтинги, составленные на основе комбинаций различных индикаторов, дают более объективную оценку, чем официальный статус и сформированные общественные представления об истории и репутации учебного заведения [14]. Первым мировым рейтингом университетов считается так называемый Шанхайский рейтинг, официально именуемый «Академический рейтинг университетов мира» (ARWU). В его методологии используется шесть объективных показателей для ранжирования мировых университетов, включая число выпускников и сотрудников, получивших Нобелевские премии и медали по Филдсу, высокоцитируемых исследователей, отобранных «Clarivate Analytics», статей, опубликованных в журналах «Nature» и «Science», статей, индексируемых в индексе научного цитирования (SCIE) и индексе цитирования по социальным наукам (SSCI), а также показатели на душу населения университета [13].

Мировой рейтинг «Тhe Times Higher Education World University Rankings» оценивает исследовательские университеты по 13 показателям, сгруппированным по 5 направлениям: преподавание (среда обучения); научные исследования (объем, доход и репутация); цитирование (влияние исследований); международные перспективы (персонал, студенты и научные проекты) и отраслевые доходы (передача знаний) [28]. 
«Quacquarelli Symonds World University Rankings» - еще один рейтинг, составляющий мировую тройку, - использует ряд академических показателей для измерения: репутацию учебного заведения, индекс цитирования публикаций исследователей и долю персонала с учеными степенями Ph.Ds. [29].

Вместе с тем три самых влиятельных глобальных рейтинга университетов «Shanghai Ranking Consultancy» (SRC), «Times Higher Education» (THE) и «Quacquarelli Symonds» (QS), - наряду с другими глобальными рейтингами, прежде всего измеряют достижения в исследованиях вузов, а не в качестве обучения студентов. Они критикуют за доминирование наукометрии над образованием, которое сущностно важно для университета [24], и ставят под сомнение обоснованность используемых индикаторов [14].

Отечественные рейтинги фактически не востребованы ни надзорными государственными структурами, ни общественными организациями. Парадокс заключается в том, что, несмотря на это, ведущие российские вузы ведут жесткую борьбу за лучшие места в них [4].

Российские исследователи разработали модельную методологию многомерного ранжирования вузов России, которая позволяет оценить их с учетом разнообразных миссий и функций. Она определяет принципы, критерии и индикаторы построения модели ранжирования вузов на мировом и национальном уровнях, а ее аналитическая рамка служит для анализа и сравнения международных и российских систем рейтингования [7]. В методологии выделяется 5 направлений оценки деятельности вузов: научно-исследовательская и международная деятельность, обучение и преподавание, трансфер знаний и взаимодействие с регионом [6].

Каждый рейтинг строится, исходя из избранной модели университета, которая служит рамкой оценки существующей системы высшего образования [2]. Так, Московский международный рейтинг (МосMР) «Три миссии университета» ориентирован на создание конкурентной среды посредством взаимодействия вузов с обществом: образование для образования и интенсификации научных разработок, их социальный эффект и способность отвечать на вызовы времени, учитывая стра- новые, региональные и глобальные контексты образовательной среды [5].

При составлении профильных рейтингов преследуется цель отразить актуальные аспекты развития университетов, исходя из возникающих приоритетов развития экономики и политической конъюнктуры. Например, методология рейтинга «Reuters World Top 100: инновационных университетов» построена на измерении 10 индикаторов. Показатели учитывают академические работы, которые указывают на проводимые в вузе фундаментальные исследования, а также патентные заявки, демонстрирующие заинтересованность учреждения в защите и коммерциализации инноваций [23].

Однако ни один из существующих рейтингов не дает исчерпывающего представления об уровне продвинутости университетов в цифровизации. Отчасти эту функцию выполняет рейтинг «Webometrics», также известный как веб-рейтинг вузов, проводимый Испанским национальным исследовательским советом. Он построен на основании базы данных более чем 20000 вузов, но в соответствии с их веб-присутствием (оценка научного содержания, видимости и влияния университетов в Интернете) предоставляет информацию об около 12000 таких учреждений. Ранжирование основано на составном показателе, который включает как объем веб-контента, так и видимость, влияние веб-публикаций в зависимости от количества внешних ссылок. Целью рейтинга является улучшение присутствия университетов и научно-исследовательских институтов в Интернете и содействие публикации научных результатов в открытом доступе. Несмотря на то, что глобальная сеть один из наиболее актуальных инструментов научной коммуникации, до сих пор эти показатели редко используются для оценки результатов научных исследований и академической успеваемости вузов [25].

Сегодня в центре внимания ученых - переход от традиционной к цифровой модели обучения [26]. Внедрение цифровых технологий порождает для образования новые вызовы и возможности. Исследователи исходят из того, что система образования должна обеспечивать обществу уверенный переход в цифровую эпоху, ориентированный на рост произво- 
дительности, новые типы труда, потребности человека. Цифровые технологии рассматриваются не только как инструмент, но и как среда существования. Их применение формирует у студентов компетенции, нацеленные на будущее [8].

В отдельных работах цифровизация университетов изучается в ракурсе готовности российского высшего образования к цифровой экономике (см., например: [3]). В данном исследовании используется методология, применяемая Мировым банком. Она составляет общую оценку из 5 статистических индикаторов: 1) применение ИТ в учебном процессе; 2) подготовка педагогических кадров к использованию ИТ в образовании; 3) информатизация управления образованием; 4) информационная инфраструктура высшего образования; 5) нормативно-правовое обеспечение цифровизации образования [15].

Университеты разрабатывают стратегии цифровизации в ответ на массовый переход к использованию новых технологий, но отсутствие общего видения приводит к неэффективному внедрению последних. Успешность внедрения цифровых технологий в вузах зависит от вложений в обучение цифровым навыкам персонала, ученых и студентов, а также их взаимодействие с сетями поддержки [27].

Исследователи считают, что каждое учреждение высшего образования должно пройти цифровую трансформацию, которая заключается во внедрении ИТ-решений, гибких и бесшовных процессов управления, изменениях корпоративной культуры и организационной структуры. Переход к созданию цифрового университета обусловлен несколькими факторами: 1) использованием целевой аудиторией - digital natives - цифровых коммуникаций; 2) ростом конкуренции между ведущими университетами, осваивающими новые технологии; 3) необходимостью оптимизации управления внутренних процессов для увеличения эффективности взаимодействия подразделений. Среди цифровых инноваций в образовании исследователи отмечают расширение онлайн-обучения в виде смешанных форм обучения (blended learning) и онлайн-курсов MOOC (Massive online open course). Сопутствующим направлением цифровизации образования стало развитие цифровых библиотек и кампусов университетов. Расширение онлайн-обучения показывает ежегодное удвоение доступных онлайн-курсов [10]. Исследователи отмечают, что следствием цифровизация стало изменение содержания образования и его организации, что сказывается на позиционировании как университетов, так и преподавателей [11]. Современные цифровые технологии дают новые инструменты для развития вузов и других образовательных учреждений во всем мире. Цифровизация обеспечивает возможности для обмена накопленным опытом и знаниями.

Появление растущего онлайн-сегмента образовательных услуг может полностью изменить ландшафт данной сферы: кроме ежегодного удвоения численности предлагаемых курсов и количества слушателей, прогнозируемая консолидированная выручка рынка МООС увеличится, по некоторым оценкам, более чем в 5 раз в 2020 году.

Вопросы, стоящие перед университетами, сводятся к выбору стратегии дальнейшего развития и направления, на котором планируется сфокусироваться. Очевидно, что уже сейчас следует разрабатывать программу цифровой трансформации для перехода в будущем к конкурентной образовательной и научно-исследовательской модели [10].

Методы и материалы. На основе анализа существующих рейтингов и результатов комплекса профильных авторских исследований была создана методика разработки системы рейтингования уровня и качества ицифровизации проиесса образования в вузах РФ.

Построение рейтинга базировалось на комплексе предварительных процедур:

1. Когнитивное картирование 60 официальных сайтов ведущих университетов - 30 зарубежных и 30 российских - с целью определения оптимального комплекса критериев цифровизации процесса образования (отбор иностранных вузов осуществлялся на основе рейтингов «QS World University Rankings by Subject 2018 Education» и «QS World University Rankings by Subject 2019 - Economics \& Econometrics», pocсийских - на базе рейтинга "QS University Rankings: BRICS - 2018»).

2. Систематизация выявленных в ходе предварительного исследования критериев и их группировка в три блока, отражающих рас- 
смотрение цифровизацию университетов с трех позиций - абитуриентов, студентов и выпускников.

3. Обоснование перспективности привлечения математического аппарата - метода сводных показателей (далее - МСП) [1-6; 12; 17-22] - для расчета уровня цифровизации вузов. Сущность МСП можно вербально определить как свертку многих оценок анализируемого объекта, проводимых по различным критериям, в одну сводную оценку, синтезирующую отдельные показатели качества объекта. Так, данный метод позволяет оценивать различные характеристики ценности, эффективности и качества объектов, имеющих существенное количество различных параметров.

Упрощенную схему построения сводного показателя $Q$ некоторого исследуемого объекта можно представить в виде последовательности шагов [1]:

0. Формируется вектор $x=\left(x_{1}, \ldots, x_{n}\right)$ исходных характеристик, каждая из которых необходима, а все они вместе достаточны для полного, всестороннего оценивания исследуемого качества объекта.

1. Формируется вектор $q=\left(q_{1}, \ldots, q_{m}\right)$ отдельных показателей, представляющих собой функции $q_{i}(x), i=1, \ldots, m$ вектора исходных характеристик $x=\left(x_{1}, \ldots, x_{n}\right)$ и оценивающих различные аспекты исследуемого объекта с использованием $m$ различных критериев. В простейшем случае каждый отдельный показатель $q_{i}$ является функцией одной исходной характеристики $x_{i}: q_{i}=q_{i}\left(x_{i}\right), i=1, \ldots, m=n$.

2 . Выбирается вид синтезирующей функции $Q(q)$, сопоставляющей вектор отдельных показателей $q=\left(q_{1}, \ldots, q_{m}\right)$ и сводную оценку $Q$ (значение сводного показателя $Q=Q(q)$ ), характеризующую исследуемый объект в целом. При этом предполагается, что синтезирующая функция $Q(q)$ зависит от вектора $w=\left(w_{1}, \ldots, w_{m}\right)$ неотрицательных параметров $w_{1}, \ldots, w_{m}$, определяющих значимость (важность, весомость, влиятельность и т. Д.) отдельных показателей $q_{1}, \ldots, q_{m}$ соответственно для сводной оценки $Q: Q=Q(q)=Q(q ; w)$.

3. Определяется значение вектора параметров $w=\left(w_{1}, \ldots, w_{m}\right), w_{i} \geq 0$, интерпретируемых как весовые коэффициенты («веса»), задающие степени влияния отдельных показателей $q_{1}, \ldots, q_{m}$ на сводную оценку $Q$.
С установлением основной схемы построения сводного показателя оценки исследуемого параметра может быть осуществлен переход к построению конкретных критериев оценки.

Анализ. Представим описание метода расчета уровня и качества цифровизации образования в университетах.

В первую очередь предлагается рассмотреть критерии оценки уровня и качества цифровизации процесса сопровождения вузами абитуриентов. Такими критериями могут быть:

1. Наличие / отсутствие на сайте вуза профориентационного онлайн-тестирования, помогающего в выборе специальности / образовательной программы (онлайн-навигатор абитуриента).

2. Коэффициент оцифрованных мастерклассов, тренингов для школьников, размещенных на сайте вуза (относительный показатель числа подразделений (кафедр), предлагающих такие информационные ресурсы, количества ресурсов (имеют больше 1 просмотра), общего числа подразделений (кафедр) вуза и общего количества ресурсов).

3. Наличие / отсутствие на сайте вуза информации для абитуриентов о профессиональных, социальных и прочих проектах, peaлизуемых в вузе или при его поддержке, и возможность участия в их реализации, в том числе через онлайн-регистрацию, до поступления в вуз.

4. Наличие / отсутствие доступа школьников к библиотечным электронным ресурсам университета в случае их участия в вузовских образовательных программах для школьников.

5. Наличие / отсутствие на сайте вуза личного кабинета абитуриента, позволяющего подать документы на поступление онлайн.

6. Относительный показатель уровня цифрового представления профессорско-педагогического состава (далее - ППС) на сайте вуза: отношение заполненных персональных страниц преподавателей, содержащих информацию об их научной деятельности, краткий список публикаций, аннотации курсов, которые они ведут, и контактные данные, к общему числу ППС вуза.

7. Наличие / отсутствие научных мероприятий для школьников (конференций, мастерклассов) с возможностью онлайн-регистрации. 
8. Относительный показатель количества онлайн-трансляций научных мероприятий для школьников (конференций, мастер-классов) к общему количеству таких мероприятий.

9. Наличие / отсутствие мероприятий, проводимых вузом и направленных на популяризацию науки с освещением мероприятия (онлайн-регистрация, онлайн-трансляция) на сайте вуза.

10. Качество образовательных программ для школьников (не подготовительные курсы). Относительный показатель количества обучающихся на подобных курсах в год, числа кафедр, предлагающих такие курсы, общего количества мест, доступных для поступления, и, наконец, общего количества кафедр.

11. Наличие / отсутствие официальных групп вуза в социальных сетях. Под официальной группой понимается группа, аффилированность которой с университетом подтверждена его руководством, а ссылка на нее размещена на сайте данного учреждения.

Для расчета оценки уровня цифровизации процесса сопровождения вузами абитуриентов предлагается использовать следующую формулу:

$$
A=\frac{k_{1}+k_{2}+\ldots+k_{11}}{N} .
$$

В ее числителе значатся критерии, которые были описаны выше, в знаменателе - нормирующий показатель. Значение нормирующего показателя будет представлено позднее.

Рассмотрим предлагаемую методику расчета каждого критерия.

$k_{1}=\{0,1\}$. Критерий принимает два значения: наличие такой -1 , ее отсутствие -0 .

$$
k_{2}=\frac{n_{1}+m_{1}}{n_{\text {общ }}+m_{\text {общ }}},
$$

где $n_{1}$ - число подразделений (кафедр) вуза, предлагающих оцифрованные мастер-классы и тренинги для школьников, $m_{1}-$ количество размещенных ресурсов, имеющих больше 1 просмотра, $n_{\text {общ }}-$ общее число подразделений (кафедр) вуза, $m_{\text {общ }}$ общее количество ресурсов.

$$
k_{3}=\{0 ; 0,5 ; 1\} . \text { Критерий принимает три }
$$
значения: на сайте вуза присутствует такая информация, существует возможность онлайн-регистрации на такие мероприятия -1 ; на сайте вуза присутствует эта информация, но отсутствует возможность онлайн-регистрации на такие мероприятия - 0,5 ; информация на сайте вуза отсутствует - 0 .

$k_{4}=\{0,1\}$. Критерий принимает два значения: наличие такой возможности -1 , ее отсутствие -0 .

$k_{5}=\{0,1\}$. Критерий принимает два значения: наличие такой возможности -1 , ее отсутствие -0 .

$$
k_{6}=\frac{n_{1}}{n_{\text {общ }}},
$$

где $n_{1}$ - количество заполненных персональных страниц преподавателей, содержащих информацию об их научной деятельности, краткий список публикаций, аннотации курсов, которые они ведут, и контактные данные, $n_{\text {общ }}$ - общее число преподавателей вуза.

$$
k_{7}=\{0 ; 0,5 ; 1\} . \text { Критерий принимает три }
$$
значения: на сайте вуза присутствует такая информация, существует возможность онлайнрегистрации на такие мероприятия - 1 ; на сайте вуза присутствует такая информация, но отсутствует возможность онлайн-регистрации на такие мероприятия $-0,5$; информация на сайте вуза отсутствует -0 .

$$
k_{8}=\frac{n_{1}}{n_{\text {общ }}},
$$

где $n_{1}$ - количество онлайн-трансляции научных мероприятий для школьников (конференций, мастерклассов), $n_{\text {об̆ }}$ - общее количество таких мероприятий, проводимых вузов.

$k_{9}=\{0,1\}$. Критерий принимает два значения: наличие такой возможности - 1 , ее отсутствие -0 .

$$
k_{10}=\frac{\frac{\sum_{i=1}^{l} n_{i}}{\sum_{j=1}^{t} n_{j} p_{j}}+\frac{m_{1}}{m_{\text {общ }}},}{2},
$$

где $n_{i}$ - количество школьников, обучающихся на $i$ образовательной программе (не подготовительные курсы), предлагаемой вузом, суммарно в течение года, $l$ - общее количество образовательных программ, $n_{j}-$ количество мест в $j$ образовательной программе для школьников, $p_{j}$ - количество проведенных $j$ образовательных программ для школьников в течение года. Расчетный параметр 


$$
\frac{\sum_{i=1}^{l} n_{i}}{\sum_{j=1}^{t} n_{j} p_{j}}
$$

показывает востребованность образовательных программ для школьников, предлагаемых вузом. $m_{1}$ - количество подразделений (кафедр) вуза, разработавших образовательные программы для школьников, $m_{\text {общ }}-$ общее число подразделений (кафедр) вуза.

$k_{11}=\{0,1\}$. Критерий принимает два значения: наличие официальных групп - 1, их отсутствие -0 .

При этом предлагается рассмотреть дополнительные веса, которые могут быть использованы для отдельных критериев. Эти веса способны выделять более важные критерии, чтобы их вклад в итоговую оценку рейтинга цифровизации вуза был более весомым. Дополнительные веса, в случае необходимости, также позволят сместить акцент оценки с одних критериев на другие. В настоящей статье предлагается рассмотреть веса критериев, нацеленные на удобство школьников при работе с сайтом вуза, а также их приобщение к научным проектам, которыми занимается вуз (табл. 1).

После ввода весов в представленную формулу расчета оценки уровня цифровизации процесса сопровождения вузами абитуриентов может быть проведен расчет нормирующего показателя, используемого в знаменателе. В рассматриваемом случае данный показатель $N=15$.

Перейдем к анализу критериев оценки уровня и качества цифровизации процесса обучения и поддержки научной деятельности студентов в вузах. Могут быть выделены следующие критерии:

1. Наличие / отсутствие доступа студентов к библиотечным электронным ресурсам вуза и внешним зарубежным электронным базам данных (SCOPUS, WoS и т. д.).

2. Наличие / отсутствие личного кабинета студента на сайте вуза.
3. Коэффициент представленности в личном кабинете студента учебно-методических материалов курсов, посещаемых студентом, относительно общего количества курсов, посещаемых студентом.

4. Наличие / отсутствие связи «расписание - аннотация курса» на сайте вуза. Под аннотацией курса понимается краткая текстовая аннотация / вводная лекция (видео- и аудиоматериал).

5. Наличие / отсутствие возможности загрузки отчетных материалов по курсу в личном кабинете учащегося, не включая функционал загрузки выпускных квалификационных работ, в том числе с целью проверки на антиплагиат.

6. Коэффициент применения e-learning и ИКТ для студентов в образовательных курсах: отношение количества курсов с применением таких технологий, доступных студентам, к общему количеству курсов, доступных студентам образовательных модулей, реализующихся с применением указанных технологий.

7. Коэффициент относительного показателя возможности дистанционного прохождения курсов / модулей относительно общего количества курсов.

8. Наличие / отсутствие на сайте вуза каталога доступных стажировок, в том числе зарубежных, для студентов всех образовательных программ с информацией об участии в них студентов вуза, а также контактов лиц, отвечающих за подготовку и поддержку студентов при оформлении заявок на такие стажировки.

9. Относительный критерий представленности массовых открытых (бесплатных) курсов на платформах онлайн-обучения (например, «Coursera») и российских национальных платформах открытого образования относительно общего количества курсов в вузе.

10. Наличие / отсутствие возможностей у вуза осуществления дистанционной формы обучения.

\section{Таблица 1. Сопоставление критериев и весов}

\section{Table 1. Comparison of criteria and weights}

\begin{tabular}{|c|c|c|c|c|c|c|c|c|c|c|}
\hline$k_{1}$ & $k_{2}$ & $k_{3}$ & $k_{4}$ & $k_{5}$ & $k_{6}$ & $k_{7}$ & $k_{8}$ & $k_{9}$ & $k_{10}$ & $k_{11}$ \\
\hline 1 & 1 & 1 & 1 & 2 & 2 & 2 & 1 & 1 & 2 & 1 \\
\hline
\end{tabular}


11. Наличие / отсутствие на сайте вуза информации о проведении общефакультетских факультативов, открытых лекций, тренингов и мастер-классов.

12. Наличие / отсутствие на сайте вуза онлайн-записи на общефакультетские факультативы, открытые лекции, тренинги и мастерклассы.

13. Относительный показатель количества онлайн трансляции общефакультетских факультативов и открытых лекций на сайте вуза к общему количеству таких мероприятий. К онлайн-трансляциям также относятся трансляции отдельных лекций, мастер-классов и семинаров.

14. Относительный показатель качества контента онлайн-трансляции общефакультетских факультативов и открытых лекций на сайте вуза, выраженный через количество участников онлайн-трансляции в ротации относительно количества участников, прошедших предварительную регистрацию.

15. Относительный показатель уровня цифрового представления ППС на сайте вуза: отношение заполненных персональных страниц преподавателей, содержащих информацию об их научной деятельности, краткий список публикаций, аннотации курсов, которые они ведут, и контактные данные, к общему число ППС вуза.

16. Наличие / отсутствие научных мероприятий для студентов (научных конференций, научных школ).

17. Относительный показатель количества онлайн-трансляций научных конференций и научных школ для студентов вуза к общему количеству таких мероприятий, в том числе трансляции отдельных лекций, мастер-классов и семинаров.

18. Относительный показатель качества контента онлайн-трансляции научных конференций и научных школ для студентов вуза, выраженный через количество участников онлайн-трансляции в ротации относительно количества участников, прошедших предварительную регистрацию.

19. Наличие / отсутствие проведения ярмарок вакансий для студентов вуза.

20. Относительный показатель количества онлайн-трансляций и/или презентаций работодателей, представленных на ярмарке вакансий, к общему количеству работодателей, представленных на этой ярмарке.

21. Наличие / отсутствие на сайте вуза информации для студентов о профессиональных, социальных и прочих проектах, реализуемых в вузе или при его поддержке, и возможность участия в их реализации, в том числе через онлайн-регистрацию.

22. Наличие / отсутствие информации о мерах поддержки и стимуляции научно-образовательной деятельности студентов.

Для расчета оценки уровня цифровизации процесса обучения и поддержки научной деятельности студентов в вузах предлагается использовать формулу:

$$
S=\frac{k_{1}+k_{2}+\ldots+k_{22}}{N} .
$$

В ее числителе значатся критерии, которые были описаны выше, в знаменателе - нормирующий показатель. Значение нормирующего показателя будет представлено позднее.

Рассмотрим предлагаемую методологию расчета анализируемых критериев.

$k_{1}=\{0,1\}$. Критерий принимает два значения: наличие такой возможности -1 , ее отсутствие -0 .

$k_{2}=\{0,1\}$. Критерий принимает два значения: наличие такого кабинета -1 , его отсутствие -0 .

$$
k_{3}=\frac{\sum_{i=1}^{m} \frac{n_{i}}{n_{\text {iобщ }}},}{m}
$$

где $n_{i}$ - количество курсов, имеющих электронные учебно-методические материалы, представленные в личном кабинете студента, по образовательной программе $i, n_{\text {обш }}-$ общее количество курсов по $i$ образовательной программе, $m$ - общее количество образовательных программ, реализуемых в вузе.

$k_{4}=\{0,1\}$. Критерий принимает два значения: наличие такой связи - 1 , ее отсутствие -0 .

$k_{5}=\{0,1\}$. Критерий принимает два значения: наличие такой возможности -1 , ее отсутствие -0 .

$$
k_{6}=\frac{\sum_{i=1}^{m} \frac{n_{i}}{n_{\text {iобщ }}},}{m}
$$


где $n_{i}$ - количество курсов, реализуемых с применением e-learning и ИКТ, по образовательной программе $i, n_{\text {іобщ }}$ - общее количество курсов по $i$ образовательной программе, $m$ - общее количество образовательных программ, реализуемых в вузе.

$$
k_{7}=\frac{\sum_{i=1}^{m} \frac{n_{i}}{n_{\text {іобщ }}}}{m}
$$

где $n_{i}$ - количество курсов с возможностью дистанционного прохождения по образовательной программе $i, n_{\text {iобщ }}-$ общее количество курсов по $i$ образовательной программе, $m$ - общее количество образовательных программ, реализуемых в вузе.

$$
k_{8}=\{0 ; 0,5 ; 1\} . \text { Критерий принимает три }
$$
значения: наличие возможности, а также сведений о контактных лицах, способных осуществить поддержку студентов, - 1 ; наличие информации без дополнительных сведений о контактных лицах $-0,5$; отсутствие какой-либо информации -0 .

$$
k_{9}=\frac{n}{n_{\text {общ }},}
$$

где $n$ - общее количество открытых (бесплатных) курсов на платформах онлайн-обучения, $n_{\text {общ }}$ общее количество курсов, реализуемых в вузе.

$$
k_{10}=\{0,1\} . \text { Критерий принимает два зна- }
$$
чения: наличие такой возможности -1 , ее отсутствие -0 .

$k_{11}=\{0,1\}$. Критерий принимает два значения: наличие такой информации -1 , ее отсутствие -0 .

$k_{12}=\{0,1\}$. Критерий принимает два значения: наличие такой возможности -1 , ее отсутствие -0 .

$$
k_{13}=\frac{n}{n_{\text {общ }}},
$$

где $n$ - общее количество онлайн-трансляции общефакультетских факультативов и открытых лекций на сайте вуза, $n_{\text {общ }}-$ общее количество таких мероприятий в вузе.

$$
k_{14}=\frac{\sum_{i=1}^{k} n_{i}}{\sum_{i=1}^{k} n_{\text {іобщ }}},
$$

где $n_{i}$ - количество участников $i$ онлайн-трансляции общефакультетских факультативов и открытых лекций на сайте вуза, $n_{i о б щ}-$ число участников такой онлайн-трансляции, прошедших предварительную регистрацию, $k$ - общее количество таких мероприятий, проведенных в вузе.

$$
k_{15}=\frac{n}{n_{\text {общ }}},
$$

где $n$ - общее количество заполненных персональных страниц преподавателей на сайте вуза, содержащих информацию об их научной деятельности, краткий список публикаций, аннотации курсов, которые они ведут, и контактные данные, $n_{\text {общ }}$ - общее количество ППС в вузе.

$$
k_{16}=\{0,1\} . \text { Критерий принимает два зна- }
$$
чения: наличие таких мероприятий - 1 , их отсутствие -0 .

$$
k_{17}=\frac{n}{n_{\text {общ }}}
$$

где $n$ - общее количество онлайн-трансляций научных конференций и научных школ для студентов вуза, $n_{\text {общ }}-$ общее количество таких мероприятий.

$$
k_{18}=\frac{\sum_{i=1}^{k} n_{i}}{\sum_{i=1}^{k} n_{\text {іобщ }},}
$$

где $n_{i}$ - количество участников $i$ онлайн-трансляции общефакультетских факультативов и открытых

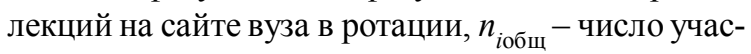
тников такой онлайн-трансляции, прошедших предварительную регистрацию, $k$ - общее количество таких мероприятий, проведенных в вузе.

$$
k_{19}=\{0,1\} . \text { Критерий принимает два зна- }
$$
чения: наличие таких ярмарок -1 , их отсутствие -0 .

$$
k_{20}=\frac{\sum_{i=1}^{k} n_{i}}{\sum_{i=1}^{k} n_{\text {общ }}^{i}},
$$

где $n_{i}$ - количество онлайн-трансляций и/или презентации работодателей, представленных на $i$ ярмарке вакансий, $n_{\text {iобщ }}$ - количество работодателей, принимающих участие в $i$ ярмарке вакансий, $k-$ общее количество таких мероприятий, проведенных в вузе.

$$
k_{21}=\{0 ; 0,5 ; 1\} . \text { Критерий принимает три }
$$

значения: наличие такой информации и нали- 
чие онлайн-записи на различные мероприятия - 1; наличие такой информации без возможности онлайн-записи на мероприятия - 0,5 ; отсутствие какой-либо информации - 0 .

$k_{22}=\{0,1\}$. Критерий принимает два значения: наличие такой информации - 1 , ее отсутствие -0 .

Помимо методики расчета критериев, предлагается рассмотреть дополнительные веса, которые могут быть сопоставлены некоторым из критериев. Введение таких весов представляется целесообразным для ранжирования критериев по важности, а также выделения тех или иных критериев в случае необходимости. Предлагаем рассмотреть веса критериев, направленные на стимуляцию научной деятельности студентов вузов (табл. 2).

После ввода весов в представленную формулу расчета оценки уровня цифровизации процесса обучения и поддержки научной деятельности студентов в вузах может быть проведен расчет нормирующего показателя, используемого в знаменателе. В рассматриваемом случае данный показатель $N=30$.

Представим критерии оценки уровня и качества цифровизации процесса сопровождения вузами выпускников. Могут быть выделены следующие критерии:

1. Наличие / отсутствие личного кабинета выпускника на сайте вуза с возможностью доступа к использованным во время получения образования учебным материалам.

2. Наличие / отсутствие информации в личном кабинете выпускника о курсах повышения квалификации с разбиением по специальностям и полученному образованию.

3. Наличие / отсутствие информации в личном кабинете выпускника о вакансиях для выпускников вуза от работодателей-партнеров с разбиением по специальностям и полученному образованию.

4. Наличие / отсутствие информации о программах поддержки вуза, инициированных группами выпускников (ассоциацией выпускников и т. д.).

5. Наличие / отсутствие на сайте вуза историй успеха выпускников с данными об их профессиональном и карьерном развитии, участии в жизни вуза.

6. Наличие / отсутствие возможности автоматизированного составления резюме для выпускника с перечислением его компетенций, полученных во время обучения в вузе.

Для расчета оценки уровня цифровизации процесса сопровождения вузами выпускников предлагается использовать следующую формулу:

$$
P=\frac{k_{1}+k_{2}+\ldots+k_{6}}{N} .
$$

В ее числителе значатся критерии, которые были описаны выше, а в знаменателе нормирующий показатель. Значение нормирующего показателя будет представлено позднее.

Рассмотрим предлагаемую методологию расчета анализируемых критериев.

$k_{1}=\{0,1\}$. Критерий принимает два значения: наличие такого кабинета -1 , его отсутствие -0 .

$k_{2}=\{0 ; 0,5 ; 1\}$. Критерий принимает три значения: наличие такой информации с разбиением по специальностям - 1 ; наличие такой информации общим перечнем; без разбиения 0,5 ; отсутствие какой-либо информации - 0 .

$k_{3}=\{0 ; 0,5 ; 1\}$. Критерий принимает три значения: наличие такой информации с разбиением по специальностям - 1 ; наличие такой информации общим перечнем, без разбиения $-0,5$; отсутствие какой-либо информации - 0 .

$k_{4}=\{0,1\}$. Критерий принимает два значения: наличие такой информации -1 , ее отсутствие -0 .

$k_{5}=\{0,1\}$. Критерий принимает два значения: наличие такой информации -1 , ее отсутствие -0 .

\section{Таблица 2. Сопоставление критериев и весов}

Table 2. Comparison of criteria and weights

\begin{tabular}{|c|c|c|c|c|c|c|c|c|c|c|}
\hline$k_{1}$ & $k_{2}$ & $k_{3}$ & $k_{4}$ & $k_{5}$ & $k_{6}$ & $k_{7}$ & $k_{8}$ & $k_{9}$ & $k_{10}$ & $k_{11}$ \\
\hline 1 & 2 & 1 & 1 & 2 & 1 & 1 & 1 & 1 & 1 & 2 \\
\hline$k_{12}$ & $k_{13}$ & $k_{14}$ & $k_{15}$ & $k_{16}$ & $k_{17}$ & $k_{18}$ & $k_{19}$ & $k_{20}$ & $k_{21}$ & $k_{22}$ \\
\hline 2 & 1 & 1 & 1 & 2 & 2 & 2 & 1 & 1 & 1 & 2 \\
\hline
\end{tabular}


$k_{6}=\{0,1\}$. Критерий принимает два значения: наличие такой возможности -1 , ее отсутствие -0 .

Помимо методики расчета критериев, предлагается рассмотреть дополнительные веса, которые могут быть сопоставлены с некоторыми из критериев. Введение таких весов представляется целесообразным для ранжирования критериев по важности, а также выделения тех или иных критериев, в случае необходимости. Предлагаем рассмотреть веса критериев, направленные на поддержание выпускников вузов в их профессиональной деятельности (табл. 3).

\section{Таблица 3. Сопоставление критериев и весов}

Table 3. Comparison of criteria and weights

\begin{tabular}{|c|c|c|c|c|c|}
\hline$k_{1}$ & $k_{2}$ & $k_{3}$ & $k_{4}$ & $k_{5}$ & $k_{6}$ \\
\hline 2 & 2 & 2 & 1 & 1 & 2 \\
\hline
\end{tabular}

После ввода весов в представленную формулу расчета оценки уровня цифровизации процесса сопровождения вузами выпускников может быть проведен расчет нормирующего показателя, используемого в знаменателе. В рассматриваемом случае данный показатель $N=10$.

С получением формулы для оценки уровней цифровизации вуза для трех категорий граждан может быть выведена общая оценка цифровизации вуза. Она может быть выражена следующим образом:

$$
C=\frac{A+S+P}{3}
$$

Результаты. Более репрезентативными, на наш взгляд, представляются три рассмотренные выше оценки для каждой из категорий. Они позволяют сделать вывод о качестве поддержки вузом абитуриентов, студентов и выпускников, а также провести коррекцию политики вуза в случае необходимости.

В данной статье к рассмотрению предложена система расчета уровня и качества цифровизации процесса образования в вузах РФ. В качестве основы расчета предложен математический аппарат - метод сводных показателей. Он позволяет получить гибкую систему расчета уровня цифровизации, учитывая при этом существенное количество различных параметров. Основой для индикаторов послужили результаты анализа передового опыта цифровизации вузовской среды ведущих зарубежных и российских университетов. Система учитывает потребности в цифровизации образовательной среды трех разных категорий граждан: абитуриентов, студентов и выпускников.

Целесообразность апробации и применения разработанной системы рейтингования уровня и качества цифровизации образования в российских университетах обусловлена следующими обстоятельствами:

- система позволяет учесть качество цифровой среды, специфичной для потребностей трех разных категорий: абитуриентов, студентов и выпускников;

- система принимает в расчет передовой опыт ведущих зарубежных и отечественных вузов в процессе цифровизации образования;

- привлечение математического аппарата объективизирует процесс расчета индекса цифровизации российских вузов, обеспечивает сопоставимость качества развития цифровых сред университетов;

- реализация рейтинга позволит обосновать систему рекомендаций администрациям вузов по повышению уровня и качества осуществляемой ими цифровизации процесса образования.

Дальнейшее исследование, как представляется, лежит в области развития и уточнения критериев уровня цифровизации вузов - разработка новых критериев, пересмотр текущих критериев и их весов. Кроме того, очевидным требованием для переработки критериев является действительная оценка уровня цифровизации вузов по их реальным параметрам. Проведение ряда экспериментов по оценке уровня цифровизации ведущих вузов РФ позволит выявить возможные недостатки разработанной системы, равно как и дополнить ее новыми параметрами, характерными для цифровизации процесса образования в России. Кроме того, на основе представленного математического аппарата может быть разработан автоматизированный комплекс расчета уровня цифровизации, позволяющий сотрудникам вузов, ответственным за данное направление, вводить необхо- 
димые параметры и сразу получать итоговую оценку уровня цифровизации вуза. Дальнейшим развитием данного инструментария может стать автоматизированная система поиска, выявления и оценки цифровых сервисов вузов с последующим автоматическим расчетом уровня цифровизации. Предполагается, что данная система оценивает как внешние источники информации (различные платформы онлайн-обучения, общедоступную информацию о вузе в Интернете и т. д.), так и может подключатся к внутренним информационным системам вуза с целью мониторинга и анализа тех или иных ресурсов, доступных абитуриентам, студентам и выпускникам.

\section{СПИСОК ЛИТЕРАТУРЫ}

1. Балацкий, Е. В. Международные рейтинги университетов: практика составления и использования / Е. В. Балацкий, Н. А. Екимова // Экономика образования. - 2012. - № 2. - С. 67-80.

2. Гайсенок, В. А. Корреляционные связи позиций вузов в международных рейтингах / В. А. Гайсенок, О. А. Наумович, В. В. Самохвал // Высшее образование в России. - 2018. - Т. 27, № 12. -С. 20-28.

3. Днепровская, Н. В. Оценка готовности российского высшего образования к цифровой экономике / Н. В. Днепровская // Статистика и экономика. -2018. - Т. 15, № 4. - С.16-28.

4. Донецкая, С. С. Берлинские принципы ранжирования российских университетов / С. С. Донецкая // ЭКО. - 2016. - № 46 (2). - C. 159-171. - DOI: http://dx.doi.org/10.30680/ECO0131-7652-2016-2-159-171.

5. Задорожнюк, И. Е. ТОП-200 вузов в четырех международных рейтингах / И. Е. Задорожнюк, Л. Ю. Коростелева, Б. К. Тебиев // Высшее образование в России. - 2019. - Т. 28, № 3. - С. 85-95. -DOI: https://doi.org/10.31992/0869-3617-2019-28-3-85-95.

6. Императивы интернационализации / отв. ред. М. В. Ларионова, О. В. Перфильева. - М. : Логос, 2013. $-420 \mathrm{c}$.

7. Ларионова, М. В. Методология сравнительного анализа международных подходов к ранжированию высших учебных заведений / М. В. Ларионова // Вестник международных организаций. 2012. - № 1 (36). - С. 34-69.

8. Никулина, Т. В. Информатизация и цифровизация образования: понятия, технологии, управление / Т. В. Никулина, Е. Б. Стариченко // Педагогическое образование в России. - 2018. - № 8. C. 107-113.
9. Переосмысливая образование. Образование как всеобщее благо? / под ред. И. Боковой. Париж : ЮНЕСКО, 2015.- С. 49-56.

10. Сидоров, Г. Цифровой университет: применение цифровых технологий в современных образовательных учреждениях / Г. Сидоров. -Электрон. текстовые дан. - Режим доступа: https://www.itweek.ru/ digitalization/article/detail.php?ID=192831 (дата обращения: 23.11.2019). - Загл. с экрана.

11. Тульчинский, Г. Л. Цифровая трансформация образования: вызовы высшей школе / Г. Л. Тульчинский // Философские науки. - 2017. № 6. - С. 121-136.

12. Хованов, Н. В. Оценка сложных объектов в условиях дефицита информации / Н. В. Хованов // Моделирование и анализ безопасности и риска в сложных системах : тр. 7-й междунар. науч. шк. (Санкт-Петербург, 4-8 сентября, 2008 г.). - СПб. : ИПМАШ РАН, 2008. - С. 18-28.

13. About Academic Ranking of World Universities. - Electronic text data. - Mode of access: http://www.shanghairanking.com/aboutarwu.html (date of access: 27.11.2019). - Title from screen.

14. Bekhradnia, B. International University Rankings: For Good or Ill? Higher Education Policy Institute / B. Bekhradnia. - Oxford : Oxuniprint, 2016. $30 \mathrm{p}$.

15. Digital Economy Concept, Trends and Visions: Towards a Future-Proof Strategy. - Electronic text data. Mode of access: http://pubdocs.worldbank.org/en/ 513361482271099284/Digital-Economy-RussiaDiscussion-paper-2016-12-20-eng.pdf (date of access: 23.11.2019). - Title from screen.

16. Hazelkorn, E. Globalization and the Reputation Race / E. Hazelkorn // Rankings and the Reshaping of Higher Education: The Battle for World Class Excellence. - N. Y. : Palgrave Macmillan, 2011. - P. 4-28.

17. Hovanov, N. The Making of Index Numbers Under Uncertainty/ N. Hovanov, Yu. Fedotov, V. Zakharov // Environmental Indices: Systems Analysis Approach. Oxford : EOLSS Publishers Co., 1999. - P. 83-99.

18. Hovanov, N. Estimating the Overall Financial Performance of Mexican Banks Using a New Method for Quantifying Subjective Information / N. Hovanov, J. Kolari // The Journal of Financial Engineering. 1998. -№ 7 (1). - P. 59-77.

19. Hovanov, N. Qualitative Information Processing in DSS ASPID-3W for Complex Objects Estimation Under Uncertainty / N. Hovanov, V. Kornikov, I. Seregin // Proceedings of the International Conference "Informatics and Control". Saint Petersburg : SPbGU, 1997. - P. 808-816.

20. Hovanov, N. Randomized Synthesis of Fuzzy Sets As a Technique for Multicriteria Decision Making Under Uncertainty/ N. Hovanov, V. Kornikov, I. Seregin 
// Proceedings of the International Conference "Fuzzy Logic and Applications". - [S. 1.] : Zichron Yaakov, 1997. - P. 281-288.

21. Hovanov, N. A Mathematical Methods System of Decision Making for Developmental Strategy Under Uncertainty/N. Hovanov, V. Kornikov, I. Tokin // Global Environmental Change. Spective of Remote Sensing and Geographic Information Systems. - New Delhi : Oxford \& IBH Publ. Co., 1995. P. 93-96.

22. Marope, P. T. M. Rankings and Accountability in Higher Education: Uses and Misuses / P. T. M. Marope, P. J. Wells, E. Hazelkorn. - Paris : Unesco, 2013. -86 p.

23. Methodology: Ranking the World's Most Innovative Universities. - Electronic text data. - Mode of access: https://www.reuters.com/most-innovativeuniversities-2016/methodology (date of access: 25.11.2019). - Title from screen.

24. Qiang, Zh. University Rankings in Perspective / Zh. Qiang. - Electronic text data. - Mode of access: https://www.insidehighered.com/blogs/ world-view/university-rankings-perspective (date of access: 27.11.2019). - Title from screen.

25. Ranking Web of Universities. - Electronic text data. - Mode of access: http://www.webometrics.info/ en/current_edition (date of access: 25.11.2019). - Title from screen.

26. Siemens, G. Preparing for the Digital University: A Review of the History and Current State of Distance, Blended, and Online Learning / G. Siemens, D. Gasevic, S. Dawson. - Electronic text data. - Mode of access: http://linkresearchlab.org/ PreparingDigitalUniversity.pdf (date of access: 25.11.2019). - Title from screen.

27. The 2018 Digital University: Staying Relevant in the Digital Age. - Electronic text data. - Mode of access: https:/www.pwc.co.uk/assets/pdf/the-2018digital-university-staying-relevant-in-the-digitalage.pdf(date of access: 23.11.2019). - Title from screen.

28. The World University Rankings 2020: methodology. - Electronic text data. - Mode of access: https://www.timeshighereducation.com/ world-university-rankings/world-universityrankings-2020-methodology (date of access: 27.11.2019). - Title from screen.

29. What is the QS World University Rankings? - Electronic text data. - Mode of access: https://www.qs.com/rankings (date of access: 27.11.2019). - Title from screen.

\section{REFERENCES}

1. Balatskiy E.V., Ekimova N.A. Mezhdunarodnye reytingi universitetov: praktika sostavleniya i ispolzovaniya [International Rankings of Universities: The Practice of Making and Using]. Ekonomika obrazovaniya, 2012, no. 2, pp. 67-80.

2. Gaysenok V.A., Naumovich O.A., Samokhval V.V. Korrelyatsionnye svyazi pozitsiy vuzov v mezhdunarodnykh reytingakh [Correlational Relationships Between Various International Rankings for Higher Education Institutions]. Vysshee obrazovanie v Rossii [Higher Education in Russia], 2018, vol. 27, no. 12, pp. 20-28.

3. Dneprovskaya N.V. Otsenka gotovnosti rossiyskogo vysshego obrazovaniya $\mathrm{k}$ tsifrovoy ekonomike [Assessment of the Readiness of the Russian Higher Education for the Digital Economy]. Statistika i ekonomika [Statistics and Economics], 2018, vol. 15, no. 4, pp. 16-28.

4. Donetskaya S.S. Berlinskie printsipy ranzhirovaniya rossiyskikh universitetov [Berlin Principles on Ranking of Russian Universities]. EKO [ECO], 2016, no. 46 (2), pp. 159-171. DOI: http:// dx.doi.org/10.30680/ECO0131-7652-2016-2-159-171.

5. Zadorozhnyuk I.E., Korosteleva L.Yu., Tebiev B.K. TOP-200 vuzov v chetyrekh mezhdunarodnykh reytingakh [Top-200 Higher Education Institutions in Four International Ratings]. Vysshee obrazovanie $v$ Rossii [Higher Education in Russia], 2019, vol. 28, no. 3, pp. 85-95. DOI: https:// doi.org/10.31992/0869-3617-2019-28-3-85-95.

6. Larionova M.V., Perfilyeva O.V., eds. Imperativy internatsionalizatsii [Imperatives of Internationalization]. Moscow, Logos Publ., 2013. $420 \mathrm{p}$.

7. Larionova M.V. Metodologiya sravnitelnogo analiza mezhdunarodnykh podkhodov $\mathrm{k}$ ranzhirovaniyu vysshikh uchebnykh zavedeniy [Methodology of Comparative Analysis of International Approaches to Ranking Higher Education Institutions]. Vestnik mezhdunarodnykh organizatsiy [International Organisations Research Journal], 2012, no. 1 (36), pp. 34-69.

8. Nikulina T.V., Starichenko E.B. Informatizatsiya i tsifrovizatsiya obrazovaniya: ponyatiya, tekhnologii, upravlenie [Information and Digital Technologies in Education: Concepts, Technologies, Management]. Pedagogicheskoe obrazovanie v Rossii [Pedagogical Education in Russia], 2018, no. 8, pp. 107-113.

9. Bokova I., ed. Pereosmyslivaya obrazovanie. Obrazovanie kak vseobshchee blago? [Rethinking Education. Is Education a Universal Good?]. Paris, UNESCO, 2015, pp. 49-56.

10. Sidorov G. Tsifrovoy universitet: primenenie tsifrovykh tekhnologiy v sovremennykh obrazovatelnykh uchrezhdeniyakh [Digital University: The Use of Digital Technology in Modern Educational Institutions]. URL: https://www.itweek.ru/digitalization/article/detail.php? ID=192831 (accessed 23 November 2019). 
11. Tulchinskiy G.L. Tsifrovaya transformatsiya obrazovaniya: vyzovy vysshey shkole [The Digital Transformation of Education: Challenges to Higher Education]. Filosofskie nauki [Russian Journal of Philosophical Sciences], 2017, no. 6, pp. 121-136.

12. Khovanov N.V. Otsenka slozhnykh obyektov $\mathrm{v}$ usloviyakh defitsita informatsii [Evaluation of Complex Objects in Conditions of Lack of Information]. Modelirovanie $i$ analiz bezopasnosti $i$ riska $v$ slozhnykh sistemakh: trudy 7-y mezhdunarodnoy nauchnoy shkoly (Sankt-Peterburg, 4-8 sentyabrya, 2008 g.) [Modeling and Analysis of Safety and Risk in Complex Systems. Proceedings of the $7^{\text {th }}$ International Scientific School. Saint Petersburg, September 4-8, 2008)]. Saint Petersburg, IPMASH RAN, 2008, pp. 18-28.

13. About Academic Ranking of World Universities. URL: http://www.shanghairanking.com/ aboutarwu.html (accessed 27 November 2019).

14. Bekhradnia B. International University Rankings: For Good or Ill? Higher Education Policy Institute. Oxford, Oxuniprint, 2016.30 p.

15. Digital Economy Concept, Trends and Visions: Towards a Future-Proof Strategy. URL: http:// pubdocs.worldbank.org/en/513361482271099284/ Digital-Economy-Russia-Discussion-paper-2016-1220-eng.pdf(accessed 23 November 2019).

16. Hazelkorn E. Globalization and the Reputation Race. Rankings and the Reshaping of Higher Education: The Battlefor World Class Excellence. New York, Palgrave Macmillan, 2011, pp. 4-28.

17. Hovanov N., Fedotov Yu., Zakharov V. The Making of Index Numbers Under Uncertainty. Environmental Indices: Systems Analysis Approach. Oxford, EOLSS Publishers Co., 1999, pp. 83-99.

18. Hovanov N., Kolari J. Estimating the Overall Financial Performance of Mexican Banks Using a New Method for Quantifying Subjective Information. The Journal of Financial Engineering, 1998, no. 7(1),pp. 59-77.

19. Hovanov N., Kornikov V., Seregin I. Qualitative Information Processing in DSS ASPID-3W for Complex Objects Estimation Under Uncertainty. Proceedings of the International Conference "Informatics and Control”. Saint Petersburg, 1997, pp. 808-816.
20. Hovanov N., Kornikov V., Seregin I. Randomized Synthesis of Fuzzy Sets as a Technique for Multicriteria Decision Making Under Uncertainty. Proceedings of the International Conference "Fuzzy Logic and Applications". [S. 1.], Zichron Yaakov, 1997, pp. 281-288.

21. Hovanov N., Kornikov V., Tokin I.A Mathematical Methods System of Decision Making for Developmental Strategy Under Uncertainty. Global Environmental Change. Spective of Remote Sensing and Geographic Information Systems. New Delhi, Oxford \& IBH Publ. Co., 1995, pp. 93-96.

22. Marope P.T.M., Wells P.J., Hazelkorn E. Rankings and Accountability in Higher Education: Uses and Misuses. Paris, Unesco, 2013. 86 p.

23. Methodology: Ranking the World's Most Innovative Universities. URL: https://www.reuters.com /most-innovative-universities-2016/methodology (accessed 25 November 2019).

24. Qiang Zh. University Rankings in Perspective. URL: https://www.insidehighered.com /blogs/world-view/university-rankings-perspective (accessed 27 November 2019).

25. Ranking Web of Universities. URL: http:// www.webometrics.info/en/current_edition (accessed 25 November 2019).

26. Siemens G., Gasevic D., Dawson S. Preparing for the Digital University: A Review of the History and Current State of Distance, Blended, and Online Learning. URL: http://linkresearchlab.org/Preparing DigitalUniversity.pdf (accessed 25 November 2019).

27. The 2018 Digital University: Staying Relevant in the Digital Age. URL: https:// www.pwc.co.uk/assets/pdf/the-2018-digital-universitystaying-relevant-in-the-digital-age.pdf (accessed 23 November 2019).

28. The World University Rankings 2020: Methodology. URL: https://www.timeshigher education.com/world-university-rankings/worlduniversity-rankings-2020-methodology (accessed 27 November 2019).

29. What is the QS World University Rankings? URL: https://www.qs.com/rankings (accessed 27 November 2019). 


\section{Information About the Authors}

Elena V. Brodovskaya, Doctor of Sciences (Politics), Professor, Department of the Political Science and Mass Communications, Financial University under the Government of the Russian Federation, Prosp. Leningradskiy, 49, 125993 Moscow, Russian Federation; Head of the Department of the SocioPolitical Research and Technology, Moscow State Pedagogical University, Malaya Pirogovskaya St., 1/1, 119991 Moscow, Russian Federation, brodovskaya@inbox.ru, https://orcid.org/0000-0001-5549-8107

Anna Yu. Dombrovskaya, Doctor of Sciences (Sociology), Associate Professor, Department of the Socio-Political Research and Technology, Moscow State Pedagogical University, Malaya Pirogovskaya St., 1/1, 119991 Moscow, Russian Federation; Professor, Department of Political Science and Mass Communications, Financial University under the Government of the Russian Federation, Prosp. Leningradskiy, 49, 125993 Moscow, Russian Federation, an-doc@yandex.ru, https://orcid.org/0000-0002-2015-2349

Roman V. Pyrma, Candidate of Sciences (Politics), Deputy Head, Associate Professor, Department of Political Science and Mass Communications, Financial University under the Government of the Russian Federation, Prosp. Leningradskiy, 49, 125993 Moscow, Russian Federation, pyrma@mail.ru, https://orcid.org/0000-0002-3413-4264

Artur A. Azarov, Candidate of Sciences (Technology), Associate Professor, Department of Political Science and Mass Communications, Financial University under the Government of the Russian Federation, Prosp. Leningradskiy, 49, 125993 Moscow, Russian Federation, artur-azarov@yandex.ru, https://orcid.org/0000-0003-3240-597X

\section{Информация об авторах}

Елена Викторовна Бродовская, доктор политических наук, профессор Департамента политологии и массовых коммуникаций, Финансовый университет при Правительстве Российской Федерации, просп. Ленинградский, 49, 125993 г. Москва, Российская Федерация; заведующая кафедрой социально-политических исследований и технологий, Московский педагогический государственный университет, ул. Малая Пироговская, 1/1, 119991 г. Москва, Российская Федерация, brodovskaya@inbox.ru, https://orcid.org/0000-0001-5549-8107

Анна Юрьевна Домбровская, доктор социологических наук, доцент кафедры социальнополитических исследований и технологий, Московский педагогический государственный университет, ул. Малая Пироговская, 1/1, 119991 г. Москва, Российская Федерация; профессор Департамента политологии и массовых коммуникаций, Финансовый университет при Правительстве Российской Федерации, просп. Ленинградский, 49, 125993 г. Москва, Российская Федерация, an-doc@yandex.ru, https://orcid.org/0000-0002-2015-2349

Роман Васильевич Пырма, кандидат политических наук, заместитель руководителя и доцент Департамента политологии и массовых коммуникаций, Финансовый университет при Правительстве Российской Федерации, просп. Ленинградский, 49, 125993 г. Москва, Российская Федерация, pyrma@mail.ru, https://orcid.org/0000-0002-3413-4264

Артур Александрович Азаров, кандидат технических наук, доцент Департамента политологии и массовых коммуникаций, Финансовый университет при Правительстве Российской Федерации, просп. Ленинградский, 49, 125993 г. Москва, Российская Федерация, artur-azarov@yandex.ru, https://orcid.org/0000-0003-3240-597X 